\title{
Recommandations pour le fonctionnement des unités de surveillance continue dans les établissements de santé*
}

\author{
Recommendations for Intermediate Care Unit in the French Hospitals
}

\author{
R. Robert - M. Beaussier - D. Pateron - C. Ecoffey $\cdot$ F. Denys $\cdot$ D. Honnart $\cdot$ B. Misset $\cdot$ J. Reignier \\ P.-F. Perrigault $\cdot$ B. Guidet $\cdot$ S. Kerever $\cdot$ P. Guiot \\ Reçu le 25 juin 2018; accepté le 5 juillet 2018 \\ (C) SRLF et Lavoisier SAS 2018
}
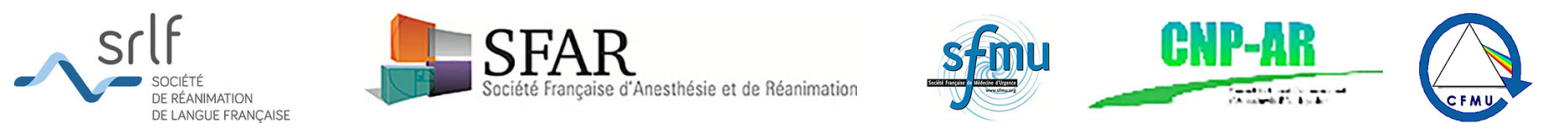

R. Robert $(\bowtie)$

Université de Poitiers, CHU de Poitiers,

médecine intensive-réanimation,

2, rue de la Milétrie, F-86021 Poitiers, France

r.robert@chu-poitiers.fr

\section{Beaussier}

Département d'anesthésie, institut mutualiste Montsouris,

42, boulevard Jourdan, F-75014 Paris, France

\section{Pateron}

Service des urgences, Sorbonne Université, CHU Saint-Antoine, Assistance publique-Hôpitaux de Paris (AP-HP),

184, rue du Faubourg-Saint-Antoine, F-75012 Paris, France

\section{Ecoffey}

Département d'anesthésie-réanimation, université Rennes-I, CHU de Rennes,

2, rue Henri-Le-Guilloux, F-35033 Rennes, France

\section{F. Denys}

Unités de surveillance continue, pôle de réanimation, université de Lille, CHRU de Lille, hôpital Roger-Salengro,

2, avenue Oscar-Lambret, F-59037 Lille, France

\section{Honnart}

Service Samu-Smur-Urgences-UOCD,

CHU de Dijon, 14, rue Paul-Gaffarel,

BP 77908, F-21079 Dijon cedex, France

\section{B. Misset}

Service de médecine intensive et réanimation, université de Rouen, CHU de Rouen,

43, rue Gambetta, F-76000 Rouen, France

\section{J. Reignier}

Service de médecine intensive et réanimation, université de Nantes, CHU de Nantes,

7, rue de Haute-Roche, F-44000 Nantes, France

\author{
P.-F. Perrigault \\ Département d'anesthésie-réanimation, \\ université de Montpellier, CHU de Montpellier, \\ 191, avenue du Doyen-Gaston-Giraud, \\ F-34090 Montpellier, France \\ B. Guidet \\ Service de réanimation médicale, Sorbonne Université, \\ CHU Saint-Antoine, AP-HP, 184, \\ rue du Faubourg-Saint-Antoine, \\ F-75012 Paris, France \\ S. Kerever \\ Direction des soins, \\ Groupe Hospitalier Saint-Louis, Lariboisière, \\ Fernand Widal, université Paris-Diderot, \\ 1, avenue Claude-Vellefaux, \\ F-75475 Paris, France \\ P. Guiot \\ Service de réanimation polyvalente, \\ centre hospitalier de Charleville-Mézières, \\ rue Savigny PRE, \\ F-08000 Charleville Mézières, France \\ CNP Médecine intensive Réanimation : R Robert, F Denys, \\ B Misset, J Reignier, B Guidet, P Guiot ; \\ CNO d'Anesthésie-Réanimation : C Ecoffey, M Beaussier, \\ PF Perrigault, S Kerever ;
}

CNP Médecine D’Urgence : D Pateron, D Honnart

* Texte élaboré par les conseils nationaux professionnels de : 1) médecine intensive-réanimation ;2) anesthésie-réanimation ; 3) médecine d'urgence et validé par la Société de réanimation en langue française (SRLF), la Société française d'anesthésieréanimation (SFAR) et la Société française de médecine d'urgence (SFMU). 
Membres du groupe de travail : René Robert, Marc Beaussier, Dominique Pateron, Bertrand Guidet, Pierre-François Perrigault Benoit Misset, Fanny Denys, Jean Reignier, Didier Honnart, Sébastien Kerver, Philippe Guiot, Claude Ecoffey.

\section{Introduction}

Les unités de soins continus (USC) sont issues du concept des unités de soins intermédiaires aux États-Unis dans les années 1980. L'objectif était d'améliorer la qualité de l'orientation des patients dès leur admission, permettant pour les patients les moins graves de limiter le coût humain, logistique et financier des séjours des patients critiques tout en garantissant une surveillance rapprochée et une prise en charge de bonne qualité. Les USC pouvaient offrir un environnement de soins « moins agressif» et plus permissif pour l'entourage familial [1,2]. Il s'agit ainsi de prendre en charge des patients ne relevant pas de la réanimation (pas d'acte de suppléance d'organe pendant plus de 24 heures) mais qui sont trop sévères pour une structure d'hospitalisation traditionnelle [1-3]. Sur ce modèle nord-américain, les lits d'USC se sont développés en France, soit intégrés ou adossés aux unités de réanimation (USC ouvertes), soit isolés et distincts des unités de réanimation (USC fermées). Les USC ont pour vocation de prendre en charge « des malades qui nécessitent, en raison de la gravité de leur état ou du traitement qui leur est appliqué, une observation clinique (incluant une surveillance rapprochée des paramètres vitaux) et biologique répétée et méthodique » [4]. Ainsi, des USC de dimension variable ont été créées dans des structures hospitalières diverses. Des règles de fonctionnement ont été suggérées par les sociétés savantes [5], des règles de valorisation de séjour ont été établies, fondées sur une liste de diagnostics essentiellement chirurgicaux, d'indices de sévérité des patients et d'actes marqueurs [6].

L'hôpital moderne est confronté à un changement de recrutement. Les patients sont plus âgés, ont plus de comorbidités, et l'on observe plus de précarité. Les contraintes démographiques, réglementaires, financières conduisent à repenser l'offre de soins avec la création des groupements hospitaliers de territoire (GHT) et le développement des prises en charge ambulatoires.

Les pouvoirs publics doivent réduire les inégalités en santé, construire des parcours de santé fluides, lisibles et sécurisés et accroître l'efficience économique. Les décrets et circulaires ont défini le champ de l'activité de réanimation et les obligations minimales de structure et d'organisation. Ils ne permettent pas de vérifier l'adéquation des structures ou de l'organisation de l'unité aux nécessités d'efficience, de qualité et de sécurité des soins. Il s'agit donc d'allouer les ressources avec équité de façon à garantir l'accès, pour tous, sans perte de chance, à des soins de qualité « proportionnés et raisonnables », avec « justesse », intégrés à l'allocation des ressources tout en préservant la qualité de vie au travail des soignants.

Dans ce contexte, la réflexion sur le secteur des soins critiques est fondamentale. Il s'agit de définir les bons niveaux d'offre de soins requis en tenant compte : de l'évolution des pratiques médicales, du niveau de complexité et/ou de rareté d'actes hautement spécialisés, des éléments structurants/ seuils, de la gradation existante, de l'organisation de la permanence des soins dans les établissements de santé. Plusieurs études ont montré le bénéfice pour les patients de telles unités [7-9]. Un travail réalisé sur 5834 patients admis en réanimation (167 réanimations dans 17 pays européens) montre que la présence d'une USC adossée à une réanimation permet de réduire la mortalité des patients [9].

Ainsi, près de 20 ans après la mise en place des premières USC, des réflexions sur la typologie de patients à y admettre (seuls 60 à $65 \%$ des patients admis bénéficient du forfait USC), sur la densité de personnels nécessaires et le dimensionnement de ces unités sont apparues. Aussi, les conseils professionnels de la médecine intensive-réanimation, de l'anesthésieréanimation $(\mathrm{AR})$ et de la médecine d'urgence ont constitué un groupe de travail afin d'analyser les conditions de fonctionnement des USC et de proposer des recommandations pour un fonctionnement optimisé de ces unités en adéquation avec les nouvelles organisations du système de santé.

Cinq champs de réflexion ont été définis : 1 . Typologie de patients 2 .

Structure des USC3.

Organisation et management paramédical 4. Organisation et management médical5.

\section{USC et GHT}

Pour chacun de ces champs, un binôme ou un trinôme du groupe de travail a proposé des recommandations pour les points paraissant clés. Le libellé de ces recommandations a été discuté et validé dans sa forme définitive par l'ensemble du groupe de travail. Chaque recommandation a été cotée par chacun des membres du groupe à l'aide d'une échelle allant de 1 (désaccord complet) à 9 (accord complet). La cotation collective a été établie selon une méthodologie dérivée de la RAND/UCLA [10] : après élimination des valeurs extrêmes (experts déviants), la médiane et l'intervalle de confiance des cotations individuelles ont été calculés. En reprenant ce qui est généralement admis, la médiane définit :

- un désaccord entre les experts lorsqu'elle est comprise entre 1 et 3 ;

- une indécision entre 4 et 6 ;

- un accord entre 7 et 9 .

Le désaccord, l'indécision ou l'accord est dit :

- « fort » si l'intervalle de confiance est situé à l'intérieur d'une des trois zones : (1-3), (4-6) ou (7-9) ; 
- « faible » si l'intervalle de confiance empiète sur deux zones.

En l'absence d'accord fort, les recommandations seront reformulées et de nouveau soumises à cotation dans l'objectif d'obtenir un meilleur consensus.

Ainsi, deux tours de cotations ont été nécessaires. Dans le document, les recommandations sont identifiées par un $\mathrm{R}$ suivi du champ de 1 à 5 .

\section{Champ no 1 : Typologie de patients}

\section{Définition des USC et place des USC dans l'hôpital : trajectoire des patients}

Les USC ne sont ni une unité de soins standard, ni une unité de réanimation. Elles font partie des unités dédiées à la prise

\section{Recommandation 1.1}

Les patients qui font l'objet d'une prise en charge en unités de soins continus (USC) doivent être sélectionnés dans un objectif de sécurité médicale et non pas d'éligibilité à un tarif. Accord fort

\section{Recommandation 1.2}

Les USC doivent être organisées de façon à prendre en charge des patients à risque de défaillance vitale nécessitant une surveillance rapprochée. Elles doivent pouvoir faire face le cas échéant à la défaillance quand elle survient et préparer le patient pour son transfert rapide en réanimation. Accord fort

\section{Recommandation 1.3}

Les moyens et les compétences développés par les USC doivent être adaptés aux qualités et aux quantités des filières de l'établissement auquel elles appartiennent dans les domaines postopératoire, d'accueil des urgences, de postréanimation et des activités « à risque » de certaines disciplines médicales. Dans les centres sans réanimation, la typologie des patients admis doit tenir compte des délais de transfert vers l'unité de réanimation correspondante. Accord fort

\section{Recommandation 1.4}

Les USC n'ont pas pour mission d'assurer la ou les suppléance (s) d'organe des patients en défaillance d'organe en dehors de la préparation d'un transfert en réanimation. Accord fort

\section{Recommandation 1.5}

Les USC ne doivent pas recevoir des patients pour engager le processus de fin de vie. Accord fort en charge des patients critiques intégrant les USC, les soins intensifs et les réanimations. Elles correspondent au terme d'intermediate care le plus souvent utilisé dans la littérature internationale $[11,12]$. Ce secteur de soins critiques peut être organisé de manière différente en fonction du type d'établissement de soins (ES) (statut, taille, localisation géographique, présence d'une réanimation dans l'établissement, présence d'une structure des urgences, recrutement à majorité chirurgicale, présence d'un Smur...).

La trajectoire des « patients critiques » admis en USC est schématisée sur la figure 1. Les modalités d'entrée sont très variables et fonction de la typologie de l'ES [7,8]. Pour les urgences, il peut s'agir aussi dans certaines régions de patients admis via le Smur ou par transfert d'autres ES.

\section{Typologie des patients}

Nous ne disposons pas à ce jour d'une photographie très précise des patients admis en USC sur le territoire national. L'enquête UNISURC (PREPS 2014) va apporter prochainement une réponse précise à cette question [13]. Les patients d'USC correspondent à des patients dont le statut médical est trop sévère et/ou la charge de soins trop élevée, rendant leur prise en charge incompatible avec les moyens alloués à une unité conventionnelle d'hospitalisation, sans justifier néanmoins d'une admission en réanimation.

Une enquête multicentrique réalisée sur 32 établissements universitaires nord-américains entre 1993 et 1998 montrait une grande similitude entre les séjours de réanimation considérés à risques faibles (recours à une thérapie de support dans moins de $10 \%$ des cas) et les séjours en USC (unité

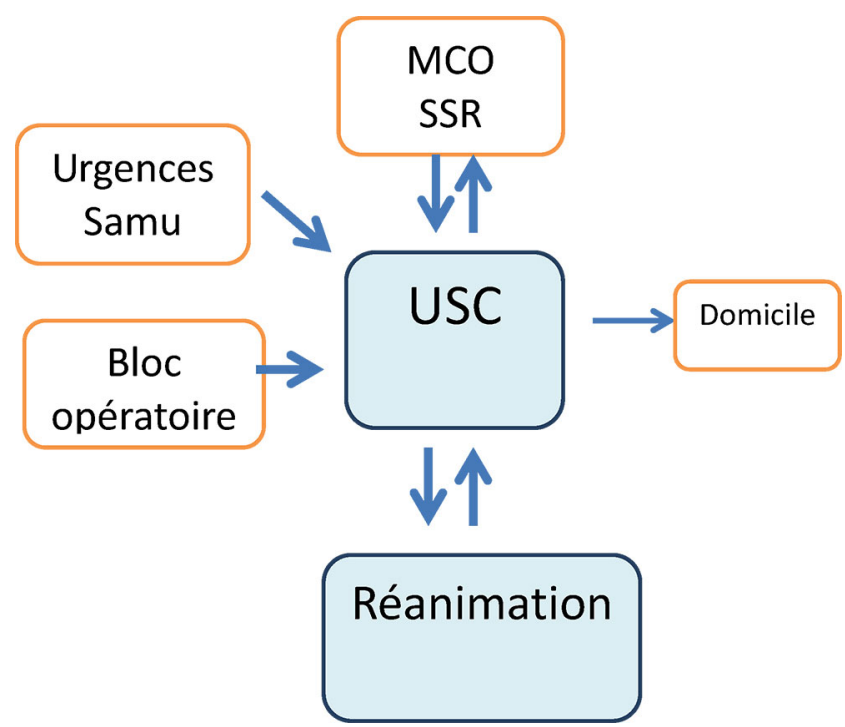

Fig. 1 Trajectoire des «patients critiques » MCO : moyens courts séjours ; SSPI : salle de soins postinterventionnelle ; USC : unité de surveillance continue ; SSR : soins de suite et rééducation 
de soins intermédiaires au sens anglo-saxon du terme) [14]. Les patients d'USC provenaient du service des urgences, du bloc opératoire, de la réanimation ou du secteur d'hospitalisation dans respectivement $37,15,17$ et $6 \%$ des cas et relevaient d'une surveillance postopératoire dans $17 \%$ des cas et d'une raison médicale dans $83 \%$ des cas. Les pathologies les plus fréquentes étaient l'angor instable (17,4\%), l'arythmie cardiaque $(8,5 \%)$, la décompensation cardiaque $(8,4 \%)$ et l'infarctus du myocarde $(4,8 \%)$. Les séjours chirurgicaux concernaient principalement la chirurgie vasculaire (endartériectomie carotidienne : $11,3 \%$, chirurgie vasculaire aortique : $7,2 \%$, pontages distaux : $4,1 \%$ et désobstruction aortofémorale : $1,8 \%$ ), la chirurgie du rachis $(10,7 \%)$, la chirurgie digestive carcinologique $(6,4 \%)$, la chirurgie du cancer pulmonaire (5\%). La présence d'au moins une comorbidité était notée dans 7,7\% des cas, et le score APACHE III moyen était de 17. La durée moyenne de séjour (DMS) de ces patients était de 3,9 jours (interquartile : [25]). Quatre-vingt-quatre pour cent d'entre eux ne faisaient l'objet d'aucune suppléance d'organe, mais le séjour était justifié par une surveillance rapprochée et des soins infirmiers lourds. $1,1 \%$ des patients étaient transférés vers la réanimation. La mortalité en USC était de $1 \%$. Même si des différences existent avec le système français, liées à l'existence de soins intensifs en particulier cardiologiques et neurovasculaires, cette typologie de patients correspond grossièrement à la population qui avait été isolée de l'enquête du benchmarking des réanimations, réalisée en France en 2011 par l'ANAP [15]. Dans cette étude, les patients avaient un IGS II médian de 30 (incluant l'âge), 18 \% de comorbidités associées, une DMS comprise entre 3,5 et 5,5 jours (moins de $2 \%$ des séjours au-delà de 28 jours) et une mortalité de 3,5\% [15]. En l'absence d'USC, ces patients sont couramment hospitalisés en réanimation où ils occupent inutilement des places pour des DMS particulièrement longues du fait de l'absence de structure adaptée d'aval.

\section{Parcours des patients et USC}

La plupart des patients admis en USC peuvent être regroupés en trois catégories principales correspondant à trois filières de soins distinctes qui paraissent représenter le plus grand nombre de patients (Fig. 1) [14]. Bien qu'il n'existe pas de donnée dans la littérature, quelle que soit la filière considérée, l'objectif est d'obtenir une durée de séjour médiane de deux à trois jours.

\section{Filière postréanimation (concept de step-down unit des Anglo-Saxons)}

Elle intéresse des patients ayant nécessité une prise en charge en réanimation et dont l'évolution favorable ne jus- tifie plus la réanimation tout en nécessitant encore une surveillance rapprochée et parfois un monitorage spécifique. En l'absence de structure d'USC, le séjour de ces patients en réanimation est prolongé avec comme conséquence une occupation non justifiée des lits et un inconfort, ou inversement ils sont transférés trop précocement vers un secteur d'hospitalisation conventionnelle avec comme conséquence une charge de soins élevée pour le personnel et une forte probabilité de survenue d'une complication [16]. Il a été montré que la survenue chez ces patients d'une complication grave en secteur d'hospitalisation conventionnelle, conduisant à la réadmission en réanimation, était associée à une mortalité importante $[17,18]$. On considère qu'environ $20 \%$ des séjours en réanimation correspondent à des patients qui pourraient relever d'une prise en charge en USC [12]. Ainsi, l'ouverture d'une USC en lien avec une réanimation permet de réduire le taux d'occupation en réanimation tout en diminuant les coûts de prise en charge pour ces patients moins sévères. Par l'effet de bascule, l'USC permet également une réduction des DMS en réanimation $[19,20]$. Il a été montré qu'il était possible de prédire de façon très fiable le besoin de soins spécifiques de réanimation à l'admission des patients, ce qui devrait permettre une meilleure sélection initiale des patients destinés préférentiellement à l'USC [12]. Toutefois, le transfert de patients de réanimation vers une USC doit être pondéré par la charge en soins inhérente à ce patient. Il faut souligner que les USC n'ont pas pour vocation d'accueillir des patients de réanimation dont la charge en soins est encore élevée. Ces patients relèveraient plus de structures de « réadaptation intensives " pour une prise en charge neuromusculaire, cognitive ou ventilatoire lourde et a priori prolongée. De telles unités que l'on pourrait qualifier de « postréanimation intensive » ou de « soins de suite et médecine physique intensive " sont rares en France et pourraient être développées sur certains sites. Ils ne font pas l'objet de ces recommandations ciblées sur des USC avec un objectif de durée de séjour brève.

\section{Filière des urgences et hospitalisation médecine- chirurgie-obstétrique (MCO) ou soins de suite rééducation (SSR)}

La deuxième filière concerne des patients admis par les urgences ou à partir d'un secteur d'hospitalisation conventionnelle consécutivement à la survenue d'une complication. On estime que $3 \%$ des patients admis aux urgences sont orientés vers les soins critiques, unités de surveillance continue (USC) ou réanimation [21]. L'enjeu est ici de dépister précocement les signes de gravité de ces patients afin de les affecter dans la structure qui leur est adaptée. Elle nécessite l'expertise de médecins qualifiés en soins critiques. 


\section{Filière postopératoire}

Le troisième groupe de patients correspond à des séjours postopératoires. La justification en est soit la survenue d'une complication anesthésique ou chirurgicale, soit la prévention d'une complication chez des patients dont la lourdeur de l'intervention et/ou l'existence de comorbidités et/ou le besoin en soins infirmiers ne permettent pas un retour direct en secteur d'hospitalisation conventionnelle. Le bénéfice d'un passage en USC pour ces patients chirurgicaux a été particulièrement bien démontré [22-26].

\section{Critères d'éligibilité pour les USC}

La sélection des patients pour l'admission en USC est fondée d'une part sur des critères objectifs de gravité et, d'autre part, sur des données statistiques permettant de sélectionner les patients à risques de complications [2,27]. On peut également considérer que la charge en soins, que ce soit dans une optique curative ou préventive, peut également à elle seule justifier un séjour en USC. Enfin, le dernier paramètre à prendre en considération est l'organisation des soins propre à chaque établissement. La présence ou non d'une réanimation à proximité de l'USC, la présence d'une salle de surveillance postinterventionnelle ouverte $\mathrm{h} 24$, un plateau technique comportant radiologie interventionnelle et endoscopie sont d'autres facteurs pouvant influer sur l'éligibilité en USC [13].

\section{Éligibilité par les critères cliniques}

De nombreuses listes ont été proposées regroupant les critères cliniques et/ou biologiques permettant de juger de la pertinence de l'admission en USC.

Les critères d'admission en USC ont été précisés dans des recommandations internationales anciennes et listées dans des ouvrages récents de réanimation (Tableau 1) [27,28]. Cependant, ces listes ne sauraient être qu'indicatives et sont modulées par la présence de structures de soins intensifs cardiologiques, d'unités neurovasculaires. De plus, l'ensemble de ces critères ne couvre pas toutes les situations. La typologie des patients et les possibilités de prise en charge évoluent, influençant les critères d'admission. Des critères organisationnels objectifs (densité de personnel, disponibilité en lits) et subjectifs jouent également un rôle dans la décision.

Concernant la filière d'admission par les urgences, un algorithme décisionnel a été proposé, permettant de réduire significativement les délais de prise en charge et d'améliorer la fluidité du parcours de soins des patients [29].

\section{Éligibilité par les scores de gravité}

Pour la chirurgie, il existe de nombreux scores permettant de prédire le risque de complications ou la mortalité postopéra- toire et qui peuvent servir à sélectionner les patients justifiant d'une poursuite de la prise en charge en USC ou en réanimation [30]. Le score ASA (American Society of Anesthesiology) est corrélé à la survenue de complications postopératoires et à la mortalité (scores ASA I, II, III et IV avec respectivement $0,1,0,7,3,5$ et $18,3 \%$ de mortalité postopératoire) [31]. Il en est de même pour le score de Charlson [31]. Ces scores (POSSUM [Physiological and Operative Severity Score for the enUmeration of Mortality and morbidity], Surgical Risk Score [SRS]) ont permis de prédire les risques de morbimortalité postopératoire afin de pouvoir mieux les prévenir et ont aidé à identifier les pathologies pouvant bénéficier de circuits spécifiques [32,33]. Cependant, la limitation principale de ces scores tient à ce que l'état de santé du patient ne suffit pas à déterminer à lui seul le risque postopératoire et doit être combiné à la lourdeur et aux éventuelles complications peropératoires de l'intervention chirurgicale si l'on veut améliorer sa sensibilité. Ils ne sont pas utilisés en routine pour l'orientation d'un patient vers une USC.

\section{Éligibilité selon les actes pratiqués et la charge de soins}

L'admission en USC peut se justifier selon les actes pratiqués et qui nécessitent soit une expertise particulière, soit entraînent une charge en soins particulièrement importante ne pouvant être délivrée dans les secteurs d'hospitalisation conventionnelle. Il existe des listes d'actes répondant à ces critères [16,34]. La ventilation non invasive (VNI) pour des situations de détresse respiratoire aiguë et l'utilisation de catécholamines (sauf exception) ne relèvent pas d'une prise en charge USC. Ces deux types de prise en charge servent souvent de curseur pour l'admission d'un patient critique en USC ou en réanimation. De nombreux outils ont été évalués par le passé pour évaluer la charge en soins. Le plus connu est le score TISS, dont une forme simplifiée, le TISS-28, a été appliquée à la réanimation $[35,36]$. Chaque type de soin est quantifié en unités qui correspondent à dix minutes de temps/unité. La charge globale en soins, qui correspond à l'addition des unités, est ensuite séparée en quatre classes : $<10$ unités $=$ surveillance habituelle simple, 10 à 20 unités $=$ surveillance continue ou intensive, $>20$ unités $=$ réanimation, $>40$ unités $=$ réanimation très lourde $[16,35]$. Dans la filière postopératoire, il a été montré une corrélation entre les scores de gravité, la mortalité des patients et le score TISS28 [37]. D'autres outils de calcul de charge en soins ont été développés comme le programme de recherche en nursing (PRN). La charge en soins ainsi mesurée dans les intermediate care est en moyenne de 325 minutes par patient et par jour. Ces outils sont cependant anciens et ne sont plus adaptés à l'évolution de la prise en charge des patients en situation critique. Ils n'intègrent pas les tâches en rapport avec la mobilisation des patients, la communication avec le patient 
Tableau 1 Recommandations de l'American College of Critical Care concernant les critères d'admission en unités de soin continu (USC). D'après Nasraway et al. [28]

\section{Indications à l'admission en USC}

Système cardiovasculaire

Suspicion d'infarctus du myocarde

Infarctus du myocarde avec hémodynamique stable

Troubles du rythme myocardique avec bonne tolérance hémodynamique

Entraînement électrosystolique chez un patient hémodynamiquement stable

Décompensation cardiaque modérée (classe NYHA 1/2)

Urgence hypertensive sans défaillance d'organe

Système respiratoire

Sevrage de la ventilation mécanique avec soins chroniques

Défaillance respiratoire avec trouble de l'hématose chez un patient stable nécessitant une surveillance rapprochée et/ou ventilation par CPAP

Besoin de soins respiratoires fréquents ou kinésithérapie respiratoire intense

Pathologies neurologiques

AVC stable nécessitant évaluations rapprochées ou soins de nursing fréquents

Trauma crânien aigu avec score de Glasgow $>9$ nécessitant une surveillance

Trauma crânien nécessitant nursing rapproché et soins respiratoires

Surveillance après traitement de l'anévrisme cérébral

Prise en charge d'une fuite de LCR

Surveillance d'un trauma médullaire cervical stable

Pathologie neurologique chronique nécessitant un nursing fréquent

Hémorragie méningée non sévère en attente de la sécurisation anévrismale

Dérivations du LCR en attente de shunt ventriculopéritonéal

Intoxications médicamenteuses

Indications de surveillances cardiaque/pulmonaire ou neurologique après intoxication médicamenteuse chez un patient stable

Pathologies digestives

Hémorragie digestive modérée répondant au remplissage vasculaire

Rupture de VO de faible intensité chez un patient stable

Insuffisance hépatocellulaire aiguë sans gravité vitale

Pancréatite aiguë

Système endocrinien

Décompensation diabétique ; acidocétose diabétique

Décompensation diabétique hyperosmolaire sans coma

Thyrotoxicose/coma myxœdémateux

Causes chirurgicales

Transfusion sanguine/remplissage vasculaire postopératoire chez un patient stable

Période postopératoire nécessitant des soins infirmiers rapprochés sur les 24 premières heures postopératoires (endartériectomie carotidienne, chirurgie vasculaire périphérique, neurochirurgie, transplantation rénale...)

Divers

Sepsis stable sans choc ni défaillance d'organe

Patients nécessitant un monitorage du remplissage vasculaire

Pathologies obstétricales éclampsie/prééclampsie ou autre problèmes

Soins infirmiers lourds/pansements complexes

NYHA : New York Heart Association ; CPAP : Continuous Positive Airway Pressure ; LCR : liquide céphalorachidien 
ou ses proches ni les tâches administratives qui ont pris une importance majeure.

\section{Éligibilité selon l'organisation de l'établissement}

La présence ou non d'une unité de réanimation dans l'établissement conditionne grandement les admissions en USC. En l'absence de réanimation, les recommandations communes SRLF-SFAR sur le fonctionnement des USC stipulaient déjà clairement qu'une convention devait être passée avec un établissement voisin prêt à accueillir les patients d'USC en réanimation [5]. Dans certaines structures, la présence d'une salle de surveillance postinterventionnelle (SSPI) ouverte 24 heures/24 peut permettre à un nombre important de patients une surveillance et une prise en charge adaptée sur les premières heures postopératoires, avec un bénéfice significatif sur l'incidence de complications postopératoires [38]. La fermeture d'une SSPI la nuit entraînera par conséquent un recours plus important aux lits d'USC pour ces patients.

\section{Critères actuels donnant lieu à valorisation par le forfait USC}

Les critères d'éligibilité actuels, reposant sur le forfait USC, sont complexes et parfois assez éloignés de la réalité médicale des patients pris en charge dans ces unités comme des enjeux de prévention qui occupent actuellement une grande place dans les stratégies de réduction de la mortalité postopératoire (Tableau 2).

C'est principalement sur l'aspect surveillance-prévention que les critères actuels d'éligibilité semblent les plus inadaptés. Un certain nombre de pathologies médicales justifiant une admission en USC pour des raisons cliniques ne sont pas valorisées. Le patient avec un asthme aigu sévère en est un exemple. Cela souligne la nécessité de revoir les critères d'éligibilité donnant lieu à la valorisation des séjours en USC, en actualisant la liste de pathologies médicales justifiant d'une surveillance en USC [39]. Les critères cliniques

Tableau 2 Critères actuels de valorisation des séjours en unité de surveillance continue

Admission en postréanimation (si séjour « valorisant »)

Score IGS II sans l'âge $\geq 15$

Patient admis en postopératoire d'un acte chirurgical figurant sur une liste spécifique

IGS II sans l'âge $\geq 7$ avec :

Un acte thérapeutique figurant sur une liste spécifique

Et/ou un acte diagnostique d'une liste spécifique

IGS : indice de gravité simplifié et biologiques témoignant de la sévérité d'une atteinte d'organe. De plus, de nouveaux outils de surveillance ou de prise en charge, telle l'oxygénothérapie à haut débit humidifié, devraient compléter la liste des actes marqueurs pour une hospitalisation en USC et être ainsi intégrés à cette actualisation. Ces réactualisations pourraient être proposées par un groupe de travail multiprofessionnel tous les cinq ans.

\section{Champ n⿳ 2 : Structure des USC}

\section{Nombre de lits nécessaires au fonctionnement d'une USC}

Pour les établissements ayant sur leur site une unité de réanimation, le nombre minimal de lits d'USC est égal au minimum à la moitié de celui des lits de réanimation [4].

Il s'agit bien d'une borne minimale qui doit considérer également l'activité de l'ES dans son ensemble, en particulier en prenant en compte le nombre de passages aux urgences, l'activité du bloc opératoire et le nombre de lits MCO. Par ailleurs, au niveau d'un ES, le nombre de lits d'USC doit dépendre également des possibilités d'affectation, en termes de nombre et de compétences d'infirmier(ière) diplômé(e) d'État (IDE), d'aide-soignant (AS) et de cadres de santé (voir plus loin) destinés à exercer dans l'USC et rapportés au nombre de lits ouverts.

Pour une USC située dans un site hospitalier ne comportant pas de service de réanimation, il faut tenir compte de l'activité des urgences, du bloc opératoire, du plateau technique et du nombre de lits MCO pour définir plus précisément ce nombre de lits minimal d'USC. Pour certaines structures de taille limitée, le regroupement de lits « critiques », unités de soins intensifs (USI) et USC, paraît logique.

\section{Distribution des lits de surveillance continue et de réanimation}

La structuration et l'organisation des USC en France a déjà fait l'objet de recommandations [40]. Quelle que soit leur organisation, chaque lit d'USC doit être équipé avec le matériel permettant au minimum le monitorage cardiaque, tensionnel et la saturation en $\mathrm{O}_{2}$. Toutes les chambres doivent être équipées d'arrivées d'oxygène, d'air, de vide mural et de prises de courant prioritaires. Le matériel permettant d'initier la prise en charge des patients présentant une défaillance vitale grave doit être disponible sur place, notamment chariot d'urgence, appareil de ventilation artificielle, défibrillateur [40].

Quelle que soit sa localisation, toute USC doit être liée à une unité de réanimation. Dans les ES sans réanimation sur site, les lits d'USC doivent être regroupés dans une seule et même unité de soins. Dans les ES avec réanimation sur site, 


\section{Recommandation 2.1 \\ L'existence de lits d'unités de soins continus (USC) dans une structure hospitalière devrait être soumise à autorisation. Accord fort \\ Recommandation 2.2 \\ Lorsqu'une USC est localisée dans un établissement qui com- porte un service ou une unité de réanimation, l'USC doit être placée sous la même autorité médicale et paramédicale que le service ou l'unité de réanimation. Accord fort}

\section{Recommandation 2.3}

Une USC doit avoir un projet médical et paramédical spécifique à l'USC. Accord fort

\section{Recommandation 2.4}

Lorsqu'une USC est localisée dans un établissement sans service ni unité de réanimation, sa mission et son organisation doivent être précisées et intégrées au projet médical des soins critiques du GHT dont fait partie l'établissement. Accord fort

\section{Recommandation 2.5}

Dans les centres avec unité de réanimation, les lits d'USC peuvent être soit intégrés à l'unité de réanimation, soit constituer une unité distincte qui, dans ce cas, doit être à proximité de l'unité de réanimation. Accord fort

\section{Recommandation $\mathbf{2 . 6}$}

De façon générale, dans une structure hospitalière, les lits de soins critiques doivent être le plus possible regroupés. Accord fort

\section{Recommandation $\mathbf{2 . 7}$}

En dehors de particularités liées à la géographie, la taille d'une USC ne doit pas être inférieure à six lits. Accord fort

comportant donc une USC adossée à la réanimation, deux modèles existent : les USC «mélangées ou intégrées » à la réanimation et les USC distinctes de la partie réanimation $[2,41]$. L'organisation d'un « service » selon l'un ou l'autre modèle est une question cruciale en raison de l'impact sur le fonctionnement du service qu'elle peut avoir. Les avantages et inconvénients des deux organisations sont résumés dans le tableau 3. Schématiquement, lorsque deux unités (réanima- tion et surveillance continue) sont individualisées, les patients sont affectés dans l'une ou l'autre en fonction de leur niveau de gravité et de leur projet thérapeutique. Cette organisation est très largement majoritaire en France. Lorsque les deux unités sont intégrées, ce sont les lits qui sont affectés ou «classifiés » chaque jour en fonction de l'état du patient.

\section{Organisation géographique et architecturale selon le type d'organisation}

Lorsque les deux activités sont intégrées, le service peut être organisé en sous-unités selon sa capacité totale, mais toutes les chambres ont la même organisation architecturale et le même équipement matériel.

Lorsque les deux activités sont organisées en deux secteurs distincts, l'architecture des chambres peut être différente, adaptée à des patients plus autonomes dans le secteur d'USC. Elles peuvent en particulier comporter chacune des sanitaires (douche et toilettes). Sur le plan matériel, les chambres d'USC ne sont pas équipées d'appareillage lourd, mais uniquement du moniteur et des systèmes de perfusion, contrairement aux chambres de réanimation. Cette organisation permet une disponibilité immédiate des chambres de réanimation et la moindre nécessité de lieux de stockage du gros matériel. Dans le secteur USC, elle permet de disposer de locaux plus adaptés à des patients conscients le plus souvent autonomes ou en reprise d'autonomie, augmentant ainsi leur confort. Cela n'empêche pas l'utilisation ponctuelle de tout ou partie du secteur d'USC en « mode réanimation » en cas d'afflux de patients. Pour favoriser cela, mais aussi permettre l'entraide entre les équipes et favoriser les circulations, il est préférable que les deux secteurs soient à proximité immédiate, voire en continuité, une simple porte faisant office de séparation virtuelle. Cela permet aussi de mutualiser les locaux dits « de logistique » entre les deux secteurs, comme dans une organisation où les deux activités sont mélangées.

\section{Organisation des équipes selon le type d'organisation}

Dans les deux types d'organisation, les équipes médicales et paramédicales doivent être communes aux deux activités. Dans un service avec deux secteurs dédiés, il existe néanmoins une différenciation au quotidien en deux équipes dédiées, l'une à l'USC, l'autre à la réanimation. Cela n'empêche pas, si nécessaire, que l'une des équipes apporte un renfort ponctuel à l'autre. L'affectation de personnel médical et paramédical à des patients posant des problèmes différents permet une meilleure répartition de la charge de travail, en raison de la visibilité plus immédiate des tâches à effectuer. Un autre avantage de l'organisation en secteurs différenciés 
Tableau 3 Avantages et inconvénients des organisations en secteurs individualisés ou mélangés des activités de réanimation et de surveillance continue

\begin{tabular}{|ll|}
\hline Réanimation et USC individualisées & Lits de réanimation et d'USC mélangés \\
\hline Transferts de patients d'un secteur à l'autre accrus & Souplesse/flexibilité : \\
& - Moins de transferts de patients \\
& - Augmentation plus facile du nombre de lits de réanimation \\
Changement de soignants si transfert d'une unité à l'autre & en cas d'afflux \\
Amélioration du confort des patients et des proches & Patients autonomes et conscients mélangés à des patients lourds
\end{tabular}

- Réduction du bruit

- Facilitation des visites (moins d'interactions)

- Architecture adaptée (douches et toilettes dans les chambres d'USC)

Charge en soin plus simplement répartie (malades « lourds » en réanimation)

\section{Équipement adapté et à poste}

$\rightarrow$ Chambres de réanimation immédiatement disponibles

$\rightarrow$ Réduction du stockage de gros matériel (ventilateurs +++ ) Projets clairement identifiés :

- Réanimation centrée sur la prise en charge de patients lourds

- USC

- Prise en charge « rapide » de patients « à risque » mais non défaillants

- Réhabilitation postréanimation

- Techniques « lourdes » non de suppléance : plasmaphérèses, VNI chronique...

Répartition médicale et paramédicale prédéterminée.

Collaboration plus aisée entre médecins et infirmières

Répartition complexe de la charge de travail

Dilution des ratios IDE/AS

Matériel dans chaque chambre plus « adapté » aux spécificités du patient.

Projets indifférenciés

Répartition médicale et paramédicale variant selon la charge en soins

USC : unité de surveillance continue ; VNI : ventilation non invasive ; IDE : infirmier diplômé d'État ; AS : aide-soignant

est la possibilité d'une meilleure adéquation des équipes médicales et non médicales.

\section{Fonctionnement selon le type d'organisation}

L'avantage essentiel d'une unité mixant surveillance continue et réanimation est de ne pas avoir à transférer un patient d'un secteur à un autre en fonction de son évolution. Un avantage serait ainsi de limiter la charge de travail des équipes. En fait, le nombre de transferts de patients en cas d'unités séparées dépend avant tout de la stratégie d'admission et de sortie des patients. Une stratégie favorisant des admissions préférentielles en réanimation, y compris de patients peu graves, donne une impression de plus grande « sécurité médicale », mais impose un nombre de transferts plus importants. Une stratégie visant à affecter au mieux les patients dans le secteur correspondant à leur niveau de gravité réduit considérablement ces transferts. Cette dernière stratégie est justifiée par la différenciation finalement assez facile des types de patients (cf. chapitre précédent). Un autre avantage d'une organisation en un seul secteur indifférencié serait de permettre une meilleure souplesse d'organisation, le service étant jugé plus rapidement adaptable aux flux de patients proposés. Cet avantage architectural est théorique dans la mesure où il peut être prévu dans une organisation en unités distinctes d'adapter l'équipement des chambres ponctuellement si besoin, mais que le problème essentiel est de pouvoir disposer du personnel en nombre suffisant pour accueillir un afflux de patients de réanimation.

\section{Champ $n^{0} 3$ : Organisation et management paramédical}

La création des USC vise à optimiser la gestion des lits de réanimation. L'équipe paramédicale est un acteur incontournable, et la notion d'équipe soignante est capitale pour l'efficience de ces services. Le management de ces équipes 


\section{Recommandation 3.1 \\ L'équipe paramédicale d'une unité de soins continus (USC) doit faire l'objet d'une organisation et d'un projet de soins spéci- fiques. Accord fort}

\section{Recommandation 3.2}

Les personnels paramédicaux qui exercent en USC doivent recevoir une formation d'adaptation à l'emploi spécifique aux patients à risque de défaillance vitale et leur permettant d'assurer leur prise en charge en cas d'aggravation. Accord fort

\section{Recommandation $\mathbf{3 . 3}$}

La formation des paramédicaux doit aborder les aspects techniques, les aspects de prévention et de maîtrise de la qualité, et les aspects humains et psychologiques des patients en situation de dysfonction vitale. Accord fort

\section{Recommandation 3.4}

L'effectif paramédical (IDE et AS) physiquement présents et affectés aux soins doit être au moins de 0,4 par lit ouvert. Accord fort

\section{Recommandation $\mathbf{3 . 5}$}

Dans les centres avec unité de réanimation, le planning IDE doit être mutualisé entre USC et réanimation (rotation des personnels entre réanimation et USC) afin de permettre une meilleure expertise pour la prise en charge des patients.

Dans les centres sans unité de réanimation, la mutualisation du personnel paramédical avec celui des autres soins critiques (urgences, soins intensifs) est recommandée. Accord fort

nécessite une animation (leadership), un projet et un encadrement. Dans les USC de petite taille, l'encadrement peut être partagé avec celui du service de réanimation ou d'une autre unité de prise en charge de patients critiques dans les structures sans réanimation (USI, urgences). Les personnels recrutés pour le fonctionnement d'USC doivent être capables de détecter les signes d'aggravation des patients, d'initier des mesures de réanimation permettant le transfert en réanimation dans de bonnes conditions, de prendre en charge des patients très divers médicaux, chirurgicaux et traumatisés (cf. champ 1). Un encadrement assurant l'adaptation à l'emploi doit être défini dans le projet de soins de l'USC. La liste des compétences et les critères pour l'adaptation à l'emploi établis à partir du référentiel de compétence de l'infirmière en réanimation [42] et du livret d'adaptation à l'emploi [43] sont listés dans les annexes A, B.

\section{Effectifs}

Le nombre nécessaire de personnels paramédicaux n'est pas fixé par le décret 2002-466 du 5 avril 2002 [4]. Le texte de référence SRLF-SFAR de 2005 recommande un effectif d'un IDE pour quatre patients et un AS pour quatre à six patients présents dans l'unité [5]. Un minimum d'un kinésithérapeute est nécessaire.

La densité en personnel paramédical dépend de la charge en soins imposée à l'unité, elle-même fonction de la typologie des patients. Il existe à ce jour plusieurs outils de calcul de la charge en soins fondés sur le référencement des actes, mais ces mêmes échelles ne mesurent pas ou peu la charge en soins réelle et globale des USC (cf. plus haut). Les éléments principaux liés à la charge en soins sont résumés dans le tableau 4.

Plus le nombre de lits est important, plus le turn over est grand et donc plus la charge en soins, incluant une charge administrative liée aux admissions-sorties des patients, est élevée. La gravité et la typologie des patients admis entraînent une densité de surveillance et d'actes de soins. Sont particulièrement consommateurs de soins : certaines pathologies (liste non exhaustive) : asthme aigu, coma acidocétosique, cérébrolésés, hémorragie digestive, décompensation de bronchopneumopathie obstructive (BPCO)... ; certains actes de soins : pansements chirurgicaux complexes, prise en charge de la douleur (traumatologie, postopératoire, traumatismes thoraciques), traitement des troubles métaboliques complexes (acidocétose), situations avec agitation des patients ; l'immobilisation du patient du fait d'un traumatisme cervical ou de fractures multiples, pour la mobilisation et les soins d'hygiène, la prise en charge et la préparation au transfert en réanimation chez les patients qui s'aggravent. $\mathrm{La}$

Tableau 4 Éléments associés à la charge en soins dans une USC

Nombre total de lits

Turnover important

Pansements chirurgicaux complexes

Gestes invasifs : pose de cathéters centraux, drains

thoraciques

Prise en charge de la douleur

Densité de surveillance

Densité de traitements

Prise en charge de l'agitation

Rééducation intensive

Préparation au transfert en réanimation 
prise en charge des proches pour ces patients en situation critique est également une charge de travail importante.

La typologie de patients admis en USC peut varier selon la proximité (sur le même site) ou non d'une unité de réanimation. Du fait de cette proximité, les unités sont susceptibles d'accepter des patients plus graves que dans des unités isolées. Il existe donc potentiellement une différence notable de charge en soins en fonction de la localisation de l'USC. Cela n'a jamais été mesuré à notre connaissance.

En rapport avec la charge en soins, une densité adéquate de personnel paramédical doit être affectée. Les recommandations SRLF-SFAR de 2011 étaient un ratio d'un IDE pour quatre patients et un AS pour six patients [5]. Bien que les tâches de soins IDE et AS soient bien définies, il est admis de raisonner en nombre global de personnels paramédicaux (IDE-AS) devant être affectés à une USC. Le nombre nécessaire d'AS peut varier selon les stratégies choisies dans un établissement hospitalier pour le bionettoyage du matériel, des chambres (sols, lavabos, éviers).

Le ratio de personnels paramédicaux physiquement présents et affectés aux soins par patient doit être au moins de 0,4 par lit ouvert.

L'intervention d'autres acteurs paramédicaux dans les USC doit être facilitée et adaptée aux besoins et au nombre de lits de l'unité : diététicienne, ergothérapeute, psychologue, orthophonistes.

\section{Personnel fixe ou tournant réa-USC}

Le personnel paramédical des USC est confronté aux situations d'aggravation de certains patients, à la détection des signes en rapport avec cette détérioration clinique et doit être capable d'assurer la prise en charge initiale de patients en situation critique. Cette prise en charge doit être assurée avec une densité de personnel plus basse que dans une unité de réanimation. Ce personnel doit donc être bien formé. Ainsi, pour les USC intégrées aux unités de réanimations, le problème ne se pose pas : le personnel paramédical est de facto le même. Le calcul des effectifs est fait selon les pourcentages respectifs de lits de réanimation et USC, et la mutualisation est plus aisée. Cette mutualisation est facilitée pour les USC intégrées aux unités de réanimation (voir champ $\mathrm{n}^{\circ}$ 3 ci-après). Il est par ailleurs suggéré que la rotation des équipes dans une unité où la mortalité est moindre qu'en réanimation pourrait être un facteur susceptible de diminuer l'épuisement professionnel, mais cela n'est pas démontré.

Pour les USC sans unité de réanimation dans une même structure hospitalière, une équipe paramédicale doit être dédiée à l'USC. Une mutualisation avec les autres secteurs de soins critiques, soins intensifs, urgences est recommandée. Une activité « couplée » avec le Smur est possible, mais elle doit intégrer les déplacements extérieurs liés à sa mission Smur pour des durées souvent indéterminées. Une mutualisation avec des équipes de soins conventionnels n'est pas recommandée. Des formations à la prise en charge de patients critiques doivent être prévues dans le plan de formation et organisées lors du recrutement et tout au long de l'exercice en USC.

\section{Encadrement}

Un cadre de santé est nécessaire pour une unité individualisée de plus de dix lits. Pour des unités de taille plus réduite, le cadre de santé est commun avec l'unité de réanimation ou avec un autre secteur d'activité de soins critiques dans les établissements hospitaliers sans réanimation.

\section{Autres personnels}

La présence d'un kinésithérapeute et la possibilité de faire appel à une prestation de kinésithérapie 7 jours/7 sont indispensables au fonctionnement d'une USC. La présence d'un temps de psychologue est souhaitable. Les liens avec assistant(e) social(e), diététicien, orthophoniste, psychomotricien... doivent être précisés dans le projet de service.

\section{Formation}

De nombreuses connaissances et compétences sont nécessaires pour qu'un infirmier soit capable de prendre en charge des patients de surveillance continue en assurant leur sécurité et en dispensant des soins de qualité. La procédure d'adéquation à l'emploi doit permettre aux futurs IDE d'USC d'acquérir les connaissances et les comportements indispensables à l'exercice de leur profession [43]. La formation d'adéquation à l'emploi est de facto commune à celle de la réanimation pour les USC intégrées aux unités de réanimation, qu'elles soient ouvertes ou fermées. Pour les USC distinctes de l'unité de réanimation, une formation en service de réanimation, prévue dans le projet de service, doit être effectuée avant d'intégrer l'USC. L'actualisation de cette formation doit être prévue dans le plan de formation de l'unité.

\section{Champ $n^{0} 4$ : Organisation et management médical}

L'organisation médicale et le management médical d'une USC doivent répondre aux impératifs majeurs de la sécurité de la prise en charge des patients dans le cadre d'une politique de la qualité qui doit pouvoir être évaluée. Cela suppose une définition et une clarification du parcours patient des malades hospitalisés en USC. Par ailleurs, il est indispensable dans les USC des centres universitaires et souhaitable dans les USC des centres non universitaires d'intégrer une politique de recherche clinique. L'organisation 


\section{Recommandation 4.1 \\ Les médecins exerçant en unités de soins continus (USC) doi- vent faire l'objet d'une formation initiale ou attestée à l'activité des soins critiques - par l'intermédiaire de la formation à l'anesthésie-réanimation, à la médecine intensive-réanimation ou à la médecine d'urgence. Accord fort}

\section{Recommandation 4.2}

L'équipe médicale qui prend en charge une USC, rattachée à un service ou à une unité de réanimation, doit être sous la même responsabilité que le service ou l'unité de réanimation. Accord fort

\section{Recommandation 4.3}

L'équipe médicale d'une USC d'un établissement sans unité de réanimation doit être sous la responsabilité d'un médecin qui exerce à temps majoritaire dans l'USC. Accord fort

\section{Recommandation 4.4}

Toute unité d'USC nécessite la présence d'un médecin sur site hospitalier 24 heures/24, 7 jours/7. La permanence des soins de nuit, de week-end ou de jours fériés doit être assurée par un médecin qui répond aux critères de R.4.1. Cette activité doit être mutualisée avec le service auquel l'USC est rattachée au sein du même établissement. En l'absence de réanimation dans l'établissement, la mutualisation doit se faire avec les autres unités de soins critiques. Accord fort

\section{Recommandation 4.5}

L'activité de formation continue et d'évaluation des pratiques professionnelles des praticiens exerçant dans un établissement sans service ni unité de réanimation devrait être assurée en coopération avec le réseau de soins critiques du GHT auquel appartient l'établissement. Accord fort

médicale est modulée par l'existence ou non d'une réanimation sur le même site de l'établissement hospitalier.

Il est difficile de recommander un nombre minimal de praticiens permanents exerçant au sein d'une USC. Il va dépendre du rattachement ou non de l'USC à une unité de réanimation de l'établissement. Les effectifs médicaux nécessaires au fonctionnement d'une USC doivent prendre en compte la participation à la permanence des soins, la durée des comptes épargne-temps des praticiens et le temps de formation. De manière pragmatique, un nombre de 3,5 ETP de praticiens en contrat demi-journées ou de 4,2 en contrat temps continu semble le minimum pour une unité autonome.

\section{Formation médicale}

Les maquettes de formation des DES de médecine intensiveréanimation, $\mathrm{AR}$, médecine d'urgence et des anciens DESC (diplôme d'études spécialisées complémentaires) de réanimation médicale et de médecine d'urgence autorisent de fait la prise en charge de patients relevant des USC.

Nous recommandons la valorisation de l'intérêt du travail en USC pour tous les internes s'inscrivant dans un cursus de médecine critique : diplôme d'études spécialisées d'anesthésie-réanimation (DESAR), diplôme d'études spécialisées de médecine intensive-réanimation (DESMIR), diplôme d'études spécialisées de médecine d'urgence (DESMU) ou DESC de réanimation, DESC de médecine d'urgence.

L'activité de formation continue et d'évaluation des pratiques professionnelles des praticiens exerçant dans un établissement sans service ni unité de réanimation devrait être assurée en coopération avec le réseau de soins critiques du GHT auquel appartient l'établissement.

\section{USC dans le même site d'un établissement hospitalier ayant au moins une unité de réanimation}

L'USC est sous la responsabilité d'un médecin réanimateur. La prise en charge des patients dans de telles USC doit être réalisée par des réanimateurs quel que soit leur cursus de formation. Il n'est pas recommandé d'avoir une équipe médicale dédiée uniquement à l'USC. Ces liens réanimation-USC permettent de facto d'assurer une permanence médicale des soins 24 heures/24, 7 jours/7. En période de jour (hors week-end et jours fériés), le nombre maximal de lits d'USC affectés à un praticien en charge de l'USC de huit. La nécessité d'une garde spécifique de senior et/ou d'interne dépend du nombre de lits total de réanimation et d'USC. Les modalités de la présence médicale le week-end doivent être précisées dans le projet de service.

\section{USC au sein d'un ES ne possédant pas de service de réanimation}

De nombreuses USC fonctionnent dans des ES ne comportant pas de service de réanimation, mais où existent un service d'urgences et/ou un bloc opératoire (pas de donnée chiffrée...). La démographie médicale est contrainte dans les disciplines d'AR, de médecine intensive-réanimation et de médecine d'urgence. Le fonctionnement de telles USC dépend des ressources locales de l'ES en médecins compétents en médecine critique, selon un tableau de service précis incluant la permanence des soins. Dans la plupart des situations, le médecin sera un urgentiste ou un anesthésisteréanimateur. L'USC doit bénéficier de la présence d'un médecin sur place compétent en soins critiques. L'organisation des 
hôpitaux en GHT pourrait permettre l'intervention de médecins réanimateurs AR ou MIR d'autres ES au sein de ces USC isolées, permettant de renforcer l'équipe médicale et de favoriser les liens entre USC et services de réanimation dans le GHT. Pour les patients chirurgicaux, un comanagement avec les différentes spécialités chirurgicales est souhaitable.

\section{Convention entre les USC et le(s) service(s) de réanimation}

\section{USC dans un ES comportant un service de réanimation}

Les USC occupent une place centrale entre les services de réanimation, le service des urgences et les services de soins conventionnels.

Dans le projet de service, l'articulation entre USC et le ou les service(s) de réanimation de l'ES doit être précisée afin de fluidifier les flux de patients entrant et sortant de l'USC, notamment en précisant les conditions de prise en charge par le service de réanimation des patients qui s'aggravent et de prise en charge des patients sortant de réanimation vers l'USC. Des procédures et protocoles communs aux deux services-unités faciliteront les transferts et la continuité de soins. Enfin, l'USC doit travailler en collaboration étroite avec le service des urgences et les unités d'hospitalisation conventionnelle afin là aussi de fluidifier les flux de patients.

\section{USC dans un ES ne comportant pas de service de réanimation}

Une convention doit être établie entre toute USC « isolée » et le ou les service(s) de réanimation du GHT. Ces conventions doivent être connues de la régulation du Samu et des services concernés et tenir compte de la durée des transferts d'un établissement à l'autre (existence ou non de moyens héliportés du Smur). La qualité des transports et la fluidité des transferts de patients en situation aiguë sont des éléments majeurs qui doivent être décrits dans ces conventions.

Enfin, les conventions doivent prendre en compte l'intervention de praticiens compétents en médecine critique exerçant dans l'ensemble du GHT.

\section{Champ $n^{0} 5$ : Les unités de surveillance continue dans le contexte des Organisations territoriales de santé}

\section{Justification ou logique des groupements hospitaliers de territoire (GHT)}

Les structures de soins critiques du secteur privé ne font pas partie de l'organisation en GHT, mais la réflexion du groupe

\section{Recommandation 5.1}

Les groupements hospitaliers de territoire (GHT) doivent organiser la filière de soins critiques intégrant les USC selon le volume des différentes filières de patients « à risque » de dysfonctions vitales et selon leur gravité. Accord fort

\section{Recommandation 5.2}

Dans les centres sans unité de réanimation in situ, les USC doivent établir une convention avec l'unité de réanimation au sein du GHT. Accord fort

\section{Recommandation $\mathbf{5 . 3}$}

Une USC doit avoir un projet d'unité qui précise ses missions au sein du GHT, ses objectifs et les catégories de patients qu'elle peut ou doit prendre en charge. Accord fort

\section{Recommandation 5.4}

Pour les USC isolées, il est suggéré de favoriser le partage de temps médical avec des réanimations partenaires, dans le cadre d'un " pôle de soins critiques interétablissements », afin d'éviter les problèmes d'isolement et d'attractivité. Accord fort

de travail et ses recommandations concernant les USC ne font pas de distinction particulière entre les secteurs du public et du privé. Les GHT sont en cours de constitution. Leurs modes de fonctionnement sont définis par les décrets du 27 avril 2016 et du 2 mai 2017 issus de la loi Santé de 2016 [44], dont certains sont encore en cours d'élaboration. Le ministère de la Santé a récemment diffusé un guide pour la mise en œuvre des GHT, disponible sur le site www.santegouv.fr.

Les GHT ont été définis à partir des structures hospitalières, majoritairement publiques, qui prennent en charge les patients d'un territoire géographique et sont en général centrées sur un établissement dit « support », responsable de la gestion des grandes fonctions transversales, administratives et logistiques. Les GHT sont au nombre de 135, nombre qui ne devrait pas changer substantiellement, et ont été dessinés par les agences régionales de santé après concertation avec les établissements. Tous les GHT ont un partenariat avec le ou les CHU de leur région, que celui-ci ou ceux-ci soit (soient) ou non établissement support du GHT. Chaque GHT définit ses objectifs autour du projet médical « partagé » (PMP), lequel est en cours d'élaboration pour chaque GHT, et dont nous ne savons pas encore quelle sera l'implication réelle des communautés médicales. 
Les activités de soins critiques sont une colonne vertébrale des GHT

Parmi les activités nécessaires dans chaque GHT, l'organisation des soins pour les patients en état critique a un rôle crucial dans la solidité du GHT, à la fois parce qu'elle doit garantir la sécurité des patients du territoire, de leur domicile jusqu'au lieu le plus approprié de délivrance des traitements étiologiques et symptomatiques adaptés, et parce qu'elle doit permettre d'assurer l'accueil d'urgences vitales ou soutenir une activité interventionnelle à risque de dysfonction vitale postopératoire...

Le décret sur les GHT prévoit que la prise en charge des patients en état critique repose sur des structures de nature différente qui apportent une réponse adaptée aux besoins du patient selon une graduation de la gravité clinique et organisée le plus possible en filière de soins critiques.

\section{Les structures et l'organisation territoriale des soins critiques doivent s'adapter aux particularités historiques et géographiques de chaque territoire}

Les territoires sont variés en termes géographiques (montagne, mer, plaine, urbanisation...), en termes démographiques (dispersion, âge...), sociaux (emploi, communautés...) et en structures existantes. Les rapports entre lits d'hospitalisation et démographie sont variés d'une région à l'autre et encore plus d'un territoire à l'autre. La qualité des structures hospitalières et leur attractivité pour les personnels soignants sont aussi variées. Il est donc nécessaire d'organiser les structures en réseaux de façon à ce que la population puisse bénéficier de l'ensemble des compétences en utilisant les moyens disponibles et en minimisant les risques, en particulier le risque pour les patients de ne pas être pris en charge par une équipe appropriée.

Les établissements qui disposent d'une USC « sans réanimation » doivent en préciser les raisons dans leur projet d'établissement et dans le projet médical partagé lorsqu'ils font partie d'un GHT. Ces raisons peuvent être liées à la population accueillie (activité chirurgicale, activité d'accueil des urgences) et/ou à des contraintes géographiques. Elles doivent être assorties du respect de certaines contraintes de sécurité qui assurent la permanence et la compétence des soins pour les populations ciblées par cette prise en charge ainsi que le transfert vers des structures de réanimation de référence.

\section{Le GHT doit intégrer un volet soins critiques à son projet médical partagé}

Le volet soins critiques doit comprendre la description de la filière, de la prise en charge initiale du patient à partir du domicile, du bloc, des urgences de chaque structure, jusqu'à sa réinsertion dans la vie en dehors des secteurs de soins critiques.

Les modes d'organisation et/ou conventions entre chaque structure doivent être décrits.

Les EPS de chaque GHT doivent définir les règles de sécurité de chaque structure de soins critiques en termes de compétences et de continuité de la permanence des soins.

\section{Objectifs des soins critiques}

L'objectif de l'organisation des soins critiques d'un territoire est d'assurer la sécurité maximale pour tous les habitants du territoire. Cela nécessite de considérer que l'organisation des soins critiques comporte la prise en compte des éléments suivants :

- les structures d'hospitalisation (les patients étant adressés par les services d'urgence, les Samu, les blocs opératoires ou les services d'hospitalisation conventionnelle), telles que les unités, ou services, de réanimation spécialisées ou polyvalentes (la pédiatrie étant traitée spécifiquement par les pédiatres réanimateurs), les USI et les USC ;

- les moyens de transport « secondaires » entre les différents établissements du GHT ;

- les moyens qui permettent la réalisation des examens complémentaires utilisés couramment en soins critiques ;

- les moyens qui permettent la réalisation d'interventions chirurgicales courantes, d'endoscopie ou de radiologie interventionnelle chez les patients en état critique ;

- les moyens informatiques qui permettent d'assurer un suivi administratif, médical et soignant de qualité ;

- les techniques d'évaluation de la qualité du service rendu à la population nécessitant des soins critiques ;

- les besoins en formation médicale, paramédicale et managériale des personnels qui font fonctionner les structures de soins critiques.

\section{Les USC dans les GHT}

Parmi les structures de soins critiques, les USC ont une place essentielle entre les structures d'urgence, de réanimation ou les plateaux interventionnels d'une part et les services d'hospitalisation conventionnelle ou de réhabilitation d'autre part.

La structure d'une USC peut répondre à un des schémas suivants :

- isolée d'une réanimation, c'est-à-dire sans qu'il existe une unité de réanimation dans le même site géographique hospitalier, et liée à une unité de réanimation du GHT en lien avec un Samu/Smur ;

- adossée à une réanimation, c'est-à-dire à proximité géographique (dans le même bâtiment) et dirigée par le même 
chef de service que l'unité de réanimation, dans le même site géographique hospitalier ;

- ni isolée d'une réanimation ni adossée à une réanimation, alors qu'une telle unité existe dans le même site géographique hospitalier, et liée par convention à la réanimation de l'établissement hospitalier ;

- absente malgré l'existence d'une réanimation et malgré la réglementation en vigueur. Dans ce cas, les patients nécessitant une surveillance continue sont pris en charge dans une structure inadéquate par rapport à leur niveau de gravité clinique : de réanimation, qui offre un excès de moyens, avec un tarif injustement élevé ou à l'inverse d'hospitalisation conventionnelle, avec une sécurité insuffisante ;

- établissements pavillonnaires où l'USC et la réanimation siègent dans deux pavillons séparés.

Une USC, quel que soit son format, doit avoir un projet d'unité qui précise au minimum ses missions pour le GHT, ses objectifs, les catégories de patients qu'elle peut ou doit prendre en charge et les conventions qu'elle établit avec les structures d'amont et d'aval de la filière de soins critiques, dans la perspective d'assurer la sécurité des patients du territoire.

Remerciements Au Dr Anne Veinstein (service de médecine intensive réanimation, $\mathrm{CHU}$ de Poitiers) pour sa relecture attentive du document

Remerciement particulier au Dr Patrick Blanchet (Association des réanimateurs du privé), représentant des structures de réanimation des établissements privés, qui a participé aux réunions initiales du groupe

Liens d'intérêts : les auteurs déclarent ne pas avoir de lien d'intérêt.

\section{Annexe A. Liste des actes consommateurs de temps infirmier en unités de soins continus (USC)}

Ventilation non invasive

Oxygénothérapie à haut débit

Pansement complexe

Prise en charge d'un polytraumatisé immobilisé (fracture du bassin, trauma cervical...)

Gestion de la douleur (analgésie contrôlée par voie intraveineuse, analgésie contrôlée par voie péridurale)

Pose de cathéter central, cathéter artériel (parfois nécessaire en périopératoire de chirurgie vasculaire sans défaillance hémodynamique

Pose de drain thoracique

Ponction pleurale
Surveillance rapprochée liée à la pathologie du patient Mobilisations régulières

Formalités en lien avec des entrées et des sorties fréquentes (bionettoyage, transmissions, préparation du malade, administratif)

\section{Annexe B. Adaptation à l'emploi dans une unité de soins continus (USC)}

\section{Objectif global}

À l'issue de la formation, l'infirmier diplômé d'État (IDE) est capable de prendre en charge, de manière globale, les patients de surveillance continue, en toute sécurité pour le patient et pour lui-même.

\section{Objectifs intermédiaires}

L'IDE sera capable de mobiliser ses connaissances des grands syndromes et sa compréhension des mécanismes physiopathologiques (ou de faire les recherches qui s'imposent) dans la prise en charge des patients d'USC.

L'IDE sera capable devant toute situation d'urgence de déclencher les secours adaptés et de mettre en œuvre les manœuvres et premiers gestes d'urgence.

L'IDE sera capable d'assurer l'opérationnalité de l'ensemble du matériel utilisé dans le service (monitorage, chariot d'urgence, ventilateurs...).

L'IDE sera capable, dans un souci continuel de prise en charge globale, d'appliquer les prescriptions et d'en surveiller l'efficacité, d'assurer les soins d'hygiène et de confort, de repérer et de tenir compte des besoins des patients et de leur famille. Le sens de l'observation clinique de l'IDE prendra ici une place primordiale :

- l'IDE sera capable de participer à la prévention et à la surveillance des infections nosocomiales en unité de surveillance continue ;

- l'IDE sera capable de s'inscrire dans une équipe pluridisciplinaire en connaissant les champs d'exercice de chacun ;

- l'IDE sera capable de prendre du recul, de savoir se remettre en question et de mettre à jour ses connaissances et compétences (métier en perpétuelle évolution).

\section{Parcours type intégration pour l'IDE nouvellement affecté dans le pôle de réanimation}

Accueil dans le pôle :

- effectué par le cadre supérieur de santé ou un cadre de santé ;

- présentation du pôle : différentes unités et leurs spécificités éventuelles (oxygénothérapie hyperbare, toxicologie...), population accueillie, pathologies rencontrées 
dans le pôle, organigramme médical et équipe paramédicale, horaires ;

- visite globale du pôle ;

- présentation de la procédure d'intégration.

\section{Formation en réanimation}

Tout personnel paramédical destiné à avoir une activité en USC doit recevoir une formation de réanimation. Pour les unités directement associées à une réanimation sur le même site hospitalier, cette formation initiale de réanimation est relativement facile à organiser. Pour le personnel d'USC sans réanimation sur le site, des conventions de formation dans une réanimation du groupement hospitalier de territoire (GHT) devraient être organisées. De façon dérogatoire, les formations doivent être réalisées dans les unités de soins critiques du site.

\section{Intégration à l'équipe IDE}

L'IDE sera doublé et encadré par un IDE référent pendant deux à trois semaines. Pendant cette période, le référent prendra en charge avec le nouvel IDE un nombre de patients limité, en tenant compte des objectifs à atteindre.

\section{Le(s) référent(s)}

Il sera de préférence volontaire et motivé.

Ayant de l'ancienneté dans le service.

Il sera informé par le cadre du contenu de la procédure de formation.

Il saura assurer la liaison entre les différents référents qui auront pris en charge l'IDE en formation et le cadre.

Il devra posséder des capacités pédagogiques.

Il sera capable d'assurer des cours théoriques.

\section{Contenu de la procédure de formation}

La procédure de formation permet d'appréhender les savoirs nécessaires :

- à la surveillance de la survenue potentielle d'une défaillance vitale :

- défaillance respiratoire aiguë ;

- défaillance circulatoire ;

- défaillance neurologique;

- insuffisance rénale aiguë ;

- à la prise en charge des patients spécifiques :

- postopératoires ;

- polytraumatisés ;

- isolements protecteurs et septiques ;

- caustiques ;

- pansements complexes ;
- myopathies ;

- anorexiques ;

- comportements à risque pour le patient et pour autrui (prise de toxiques, sevrage alcoolique...) ;

- à la prise en charge du patient en fin de vie et de sa famille (LAT et soins palliatifs) ;

- à la prévention du risque d'infection nosocomiale chez les patients et aux aptitudes nécessaires à développer.

\section{Évaluation}

Pour suivre l'adaptation du nouveau recruté, une évaluation à trois niveaux sera réalisée :

- évaluation sous forme d'autoévaluation faite à l'arrivée du nouveau recruté ;

- deuxième évaluation en cours de période d'adaptation à l'emploi et une synthèse à la fin de chaque semaine entre les référents et le nouveau recruté ;

- troisième évaluation à la fin de la période d'adaptation à l'emploi (date définie à la deuxième évaluation).

Les référents seront toujours présents pour former le nouveau recruté aux gestes ou techniques qu'il n'aura pas vus pendant sa période de formation.

Ces évaluations font l'objet d'un entretien entre le nouveau recruté, les référents et le cadre. Ils feront l'objet d'un compte rendu écrit remis à l'IDE et conservé par le cadre.

Pour les savoirs, l'acquisition est dite " complète » lorsque l'IDE sait faire le lien entre les notions théoriques et les pratiques du domaine concerné.

Pour les aptitudes, le niveau 3 «sait faire dans les circonstances habituelles » est requis pour valider la période d'adaptation à l'emploi.

\section{Documents à disposition}

Référentiel de compétences IDE.

Fiches techniques concernant certains soins ou techniques spécifiques.

\section{Référentiel de compétences}

\section{Accompagnement d'un patient hospitalisé (situation de détresse vitale) et ses proches}

\section{Savoirs :}

- l'infirmier connaît et comprend :

- les modalités de la communication avec un patient et ses proches dans un contexte d'hospitalisation et/ou de détresse vitale ;

- la notion de compétence du patient ou de ses représentants : aptitude du patient ou de ses représentants à 
recevoir et à comprendre les informations, à faire des choix ;

- le cadre juridique concernant la personne de confiance, le secret professionnel, l'identitovigilance et l'inventaire d'entrée ;

- les règles et procédures concernant les visites, la communication et les transmissions des informations aux proches ;

- les principales sources d'inconfort d'un patient en unité de surveillance continue ;

- les différentes familles de médicaments permettant d'assurer l'antalgie, l'anxiolyse d'un patient en surveillance continue, leur mode d'emploi, objectifs et risques ;

- les modalités d'utilisation de l'outil informatique.

\section{Compétences :}

- l'infirmier est capable :

- d'accueillir, d'accompagner et d'informer le patient et ses proches en collaboration avec l'équipe pluridisciplinaire ;

- de mobiliser les ressources humaines et d'utiliser les moyens matériels nécessaires à l'optimisation de la communication avec le patient et ses proches ;

- d'adapter ses soins aux souhaits du patient en tenant compte des objectifs des soins et de la compétence du patient, pour préserver au mieux son autonomie ;

- d'assurer la traçabilité d'un entretien en retranscrivant les éléments clés de l'échange ;

- d'identifier les représentations des patients et des proches liées à une situation de détresse vitale ;

- d'identifier les répercussions psychosociales et économiques pour les proches d'un patient hospitalisé en surveillance continue et d'apporter une réponse adaptée ;

- d'assurer le maintien du confort du patient dans l'environnement spécifique de la surveillance continue ou lors du transfert en réanimation ;

- d'identifier les besoins particuliers du patient en situation de détresse vitale et de mettre en œuvre les moyens pour y répondre ;

- de préparer l'entourage à rencontrer, dans un environnement hospitalier « agressif », le patient en situation de détresse vitale et/ou d'atteinte à son intégrité corporelle ;

- de s'assurer de la bonne compréhension par le patient et sa famille des informations transmises par le corps médical relatives à l'évolution de son état de santé en tenant compte des priorités de prise en charge thérapeutiques et en respectant les règles professionnelles en vigueur ;

- d'évaluer la douleur et l'anxiété du patient avec les moyens mis à disposition; d'adapter les soins, manipulations et de dispenser les antalgiques, anxiolytiques sur prescription médicale ;
- de respecter et de transmettre le souhait du patient hospitalisé ;

- de mettre à disposition tous les moyens pour la réhabilitation précoce (si nécessaire) du patient hospitalisé ;

- d'assurer la traçabilité de la surveillance des patients, des produits dérivés du sang et des stupéfiants, les transmissions ciblées, les notes des différents intervenants, les examens effectués sur les outils informatiques.

\section{Patient en situation d'insuffisance circulatoire}

\section{Savoirs :}

- l'infirmier comprend et connaît :

- les bases physiopathologiques, les signes cliniques et paracliniques et les principales étiologies d'une défaillance circulatoire ;

- la procédure en cas de survenue d'un arrêt circulatoire : les différents paramètres de surveillance hémodynamique et les valeurs normales des paramètres biologiques utilisés dans ce contexte ;

- les différents types de surveillance hémodynamique : indications, procédures de mise en place, limites, complications et modalités de leur utilisation ;

- les conséquences de l'état de choc sur le comportement physique et psychologique du patient et les modes de communications adaptés à la situation ;

- les différents appareils de monitorage, leurs fonctionnements et leur maintenance ;

- les différentes familles de médicaments de l'urgence et thérapeutiques de la défaillance circulatoire, leurs modes d'emploi, objectifs et risques ;

- les bases physiopathologiques, les modalités légales, la surveillance et traçabilité de la transfusion de produits et dérivés sanguins labiles.

\section{Compétences :}

- l'infirmier est capable :

- d'identifier un arrêt circulatoire, d'alerter et de débuter les gestes de réanimation ;

- d'identifier les signes cliniques d'un état de choc et de les décrire au médecin ;

- d'organiser et d'adapter la surveillance des paramètres vitaux du patient en fonction de son état;

- de mettre en œuvre les thérapeutiques de la défaillance circulatoire selon les prescriptions médicales ;

- d'utiliser les différents systèmes de monitorage hémodynamique du service ;

- de dispenser les soins de confort requis auprès d'un patient bénéficiant d'un monitorage hémodynamique en assurant le bon fonctionnement et la sécurisation des dispositifs en place et en tenant compte de l'état circulatoire du patient ; 
- d'adapter l'administration des catécholamines et sédations selon la prescription médicale en analysant l'évolution hémodynamique retransmise par le monitorage.

\section{Patient en situation de défaillance respiratoire aiguë}

\section{Savoirs :}

- l'infirmier connaît et comprend :

- les bases physiopathologiques, les signes cliniques et paracliniques, les principales étiologies d'une insuffisance respiratoire aiguë ;

- les mécanismes d'apparition et les moyens de gestion du stress et de la crise d'un patient en détresse respiratoire aiguë ;

- les modalités de prélèvement, d'acheminement et les valeurs normales des gaz du sang ;

- les différentes techniques d'oxygénothérapie, d'assistance respiratoire invasive ou non invasive et leurs paramètres de réglages et de surveillance ;

- l'infirmier connaît les différentes interfaces de l'assistance respiratoire non invasive (invasive) ;

- les procédures d'intubation;

- les procédures de gestion des voies aériennes ;

- les techniques d'optimisation des échanges gazeux.

\section{Compétences :}

- l'infirmier est capable :

- d'identifier cliniquement une défaillance respiratoire et d'en évaluer le degré de gravité en regard du diagnostic posé et de mettre en ouvre les premières mesures de mise en sécurité du patient hors assistance respiratoire ;

- de reconnaître et de transmettre une perturbation de la gazométrie ;

- d'informer le patient sur la nécessité et les modalités de l'assistance respiratoire ;

- d'assurer le confort psychologique et physique du patient sous assistance respiratoire non invasive et celui de son entourage ;

- de s'assurer du réglage des alarmes du respirateur en fonction du mode ventilatoire et des prescriptions médicales ;

- de mettre en œuvre les différentes techniques d'oxygénothérapie et d'assistance respiratoire non invasive selon les prescriptions médicales ;

- d'installer un patient sous assistance respiratoire, la surveillance et l'organisation du transfert dans un service adapté ;

- de dispenser les soins d'hygiène et de confort au patient sous assistance respiratoire en assurant la sécurité et l'efficacité de sa ventilation.

\section{Patient en situation de défaillance neurologique (agitation incluse)}

\section{Savoirs :}

- l'infirmier connaît et comprend :

- les signes, les degrés de gravité et les conséquences potentielles de troubles de la vigilance, de convulsions, d'un déficit moteur, d'un état d'agitation ;

- les situations cliniques neurologiques pouvant nécessiter la mise en œuvre immédiate de la protection des voies aériennes ;

- les éléments cliniques et les outils d'évaluation et de surveillance neurologique selon la pathologie et l'état du patient, les étiologies et facteurs d'aggravation des défaillances neurologiques ;

- les modes de communication adaptés aux patients atteints de défaillance neurologique.

\section{Compétences :}

- l'infirmier est capable :

- d'assurer une surveillance neurologique adaptée selon le rôle propre, sur prescription médicale, de transmettre et de reporter les modifications de l'état neurologique ;

- d'utiliser les échelles d'évaluation de la conscience, de l'agitation et de la douleur en vigueur dans le service ;

- d'analyser un état d'agitation, d'en rechercher les causes et de mettre en place les actions correctives selon le rôle propre et/ou le rôle collaborant ;

- d'évaluer le niveau de conscience du patient et d'adapter sa prise en charge ;

- d'évaluer et d'adapter la prise en charge de la douleur du patient à la communication altérée.

\section{Thérapeutiques, solutés, nutrition}

\section{Savoirs :}

- l'infirmier comprend et connaît :

- les différentes familles de thérapeutiques disponibles dans le service, leurs effets et mode d'action, dispensation, reconstitution (s'il y a lieu), objectifs et risques ;

- les différents solutés ;

- les besoins nutritionnels du patient « agressé »;

- les différentes voies d'administration de la nutrition, leurs avantages et inconvénients respectifs ;

- les solutés de nutrition entérale ou parentérale utilisés dans le service et leurs procédures d'administration ;

- les signes d'intolérance des différents modes de nutrition artificielle, les moyens de les détecter, de les prévenir et d'y remédier.

Aptitudes :

- l'infirmier est capable :

- d'administrer la nutrition entérale ou parentérale selon la prescription médicale ; 
- de détecter les signes d'intolérance de la nutrition entérale et d'y remédier ;

- de détecter les signes de complications liées aux dispositifs nécessaires à l'administration de la nutrition artificielle ;

- d'anticiper et d'alerter sur une possible incapacité à garantir les objectifs nutritionnels prescrits ;

- d'expliquer au patient et à ses proches le mode de nutrition retenu, ses contraintes et ses objectifs ;

- de mettre en œuvre les techniques de rééducation de la déglutition.

\section{Compétences :}

- l'infirmier gère l'administration de la nutrition entérale selon la prescription médicale en prévenant les risques d'intolérance digestive et d'inhalation ;

- l'infirmière accompagne le patient atteint d'une lésion neurologique et/ou laryngée lors de la reprise de l'alimentation orale en détectant les éventuels troubles de déglutition, en prenant appui sur les ressources liées à ses habitudes de vie et en assurant son confort et sa sécurité.

\section{Le patient en fin de vie et l'accompagnement de ses proches}

\section{Savoirs :}

- l'infirmier connaît et comprend :

- la législation française et les recommandations des sociétés savantes régissant les limitations et arrêts des thérapeutiques, et les soins palliatifs ;

- les risques (pour lui-même ?) de deuil pathologique après un décès ;

- l'outil d'aide à la décision pour la mise en place d'une procédure de limitation ou d'arrêt des thérapeutiques disponible dans le service ;

- les traitements médicamenteux ou non permettant de soulager la souffrance morale ou physique d'un patient en fin de vie;

- les possibilités d'aide des proches d'un patient en fin de vie.

\section{Compétences :}

- l'infirmier est capable :

- de participer, en collaboration avec un médecin, à l'annonce d'une évolution défavorable au patient ou à ses proches ;

- de participer aux prises de décisions relatives à la fin de vie d'un patient ;

- de participer à l'arrêt d'un traitement de suppléance dans le cadre d'une décision collégiale ;

- d'évaluer et de mettre en œuvre, en concertation avec les différents intervenants, les mesures appropriées pour assurer le confort psychologique et physique du patient en fin de vie ;
- d'accompagner les proches d'un patient en fin de vie en collaboration avec l'équipe pluridisciplinaire ;

- d'accompagner le patient et ses proches en situation de limitation des thérapeutiques et/ou de fin de vie en privilégiant le confort physique et psychologique du patient et en tenant compte des demandes du patient et de ses proches, du contexte socioculturel, spirituel et des contraintes médicales et réglementaires.

\section{Prévention du risque d'infection nosocomiale}

Savoir :

- l'infirmier connaît et comprend :

- le rôle des référents en hygiène du pôle ainsi que les protocoles validés par le comité de lutte contre les infections nosocomiales de l'hôpital ;

- les principes et modalités de prévention des infections nosocomiales ;

- les éléments cliniques de suspicion et les critères diagnostiques d'une infection nosocomiale ;

- les différentes techniques de diagnostic des infections nosocomiales utilisées dans le service.

\section{Compétences :}

- l'infirmier est capable :

- d'appliquer les mesures de prévention des infections nosocomiales chez le patient en unité de soins continus ;

- d'assurer sa protection et celle de l'environnement visà-vis du risque de contamination infectieuse lors des soins ;

- d'informer le patient et sa famille des risques et des modalités de prévention des infections nosocomiales ;

- d'identifier et de prévenir les risques infectieux liés à la ventilation mécanique (et aux voies veineuses, artérielles et sondes... ?) en appliquant les procédures et recommandations relatives à la pathologie du patient.

\section{Compétences particulières en pansements et drainages complexes}

Savoir :

- l'infirmier connaît et comprend :

- la fonction, l'installation et le positionnement des différents pansements et dispositifs de drainage, drains pleuraux, VAC, drains de Kehr, pansements complexes OHB...).

\section{Compétences :}

- l'infirmier est capable :

- d'appliquer les prescriptions et protocoles pour l'installation du patient en fonction des pansements et des dispositifs de drainage mis en place ; 
- de surveiller, de positionner le matériel en tenant compte de l'installation du patient et d'alerter en cas d'anomalie ;

- d'assurer la surveillance clinique avec l'évolution du recueil ;

- d'entretenir et d'assurer la traçabilité des dispositifs de recueil ;

- d'appliquer en suivant les prescriptions les modalités d'ablations de ces dispositifs ;

- d'assurer le maintien et le bon fonctionnement du dispositif de pansement ou de drainage, en veillant au confort du patient et à l'absence d'effet clinique délétère.

\section{Parcours type d'intégration pour l'aide-soignant (AS) nouvellement affecté dans le pôle de réanimation}

- Accueil dans le pôle :

- effectué par le cadre supérieur ou un cadre de santé ;

- présentation du pôle : population accueillie et pathologies rencontrées dans le pôle ;

- organigramme des équipes médicale et paramédicale, horaires, organisation des différents secteurs du pôle, des spécificités ;

- visite globale du pôle ;

- présentation de la procédure d'intégration.

- Intégration à l'équipe des AS :

L'AS sera encadré pendant deux à trois semaines complètes par un ou plusieurs AS afin de découvrir le déroulement de son travail.

- Objectifs :

- sensibilisation à la dynamique de prise en charge du patient par le binôme AS/IDE ;

- première approche des soins de nursing en surveillance continue ;

- découverte de la manutention des patients ;

- découverte de l'organisation logistique du service, du « circuit propre/sale » et de l'élimination des déchets.

\section{Le(s) référent(s) :}

- il sera de préférence volontaire et motivé ;

- il aura au minium deux ans d'expérience dans le pôle ;

- il sera informé par le cadre du contenu de la procédure.

\section{Évaluation :}

- pour suivre l'adaptation du nouvel AS recruté, une évaluation sera réalisée ;

- elle fait l'objet d'un entretien entre le nouveau recruté et le cadre et intègre un compte rendu écrit remis à l'AS et conservé par le cadre.

\section{Compétences à acquérir :}

- accompagner le patient dans les actes essentiels de la vie quotidienne en tenant compte de ses besoins et de son degré d'autonomie ;
- réaliser les soins adaptés à l'état clinique du patient en utilisant des techniques préventives de manutention et les règles de sécurité pour son installation et sa mobilisation ;

- toilette complète/aide à la toilette ;

- shampoing;

- rasage ;

- soins d'ongles ;

- soins de bouche ;

- soins d'oreilles ;

- prévention d'escarres ;

- noter les repères de la SNG (s'il y en a) et être capable de repérer les éventuelles anomalies ;

- éliminer les liquides physiologiques (élimination des urines et des selles chez le patient porteur de BMR/élimination des liquides d'aspiration bronchique/thoracique/drains abdominaux ou autres) [transmissions informatisées le cas échéant] ;

- peser le patient, choisir le matériel de pesée selon le degré d'autonomie du patient ;

- positionner les électrodes ECG/capteur $\mathrm{SpO}_{2}$ et tensiomètre ;

- installer le patient : connaître les diverses techniques de manutention et de positionnement du patient sur divers matelas (réglages et utilisation du matelas), du patient douloureux, défaillant neurologique, au fauteuil (avec le matériel adapté : lève malade, coussin anti-escarre...);

- mobiliser un patient : polytraumatisé, postopératoire ;

- apprécier l'état clinique du patient :

- relever la diurèse ;

- lire une TA ;

- lire une $\mathrm{SpO}_{2}$;

- savoir relever tout changement de l'état clinique : marbrures, cyanoses, tirage, dyspnée...;

- état cutané : différents stades : escarre, mycose, hématomes...;

- douleurs ;

- être capable d'alerter l'IDE ;

- établir une communication adaptée au patient et à son entourage :

- respect ;

- discrétion ;

- confidentialité ;

- secret professionnel;

- LAT ;

- communication non verbale et utilisation de supports ;

- accompagnement de la famille et des proches ;

- accompagnement d'un patient en fin de vie ;

- prise en charge d'un patient décédé et de sa famille ;

- utiliser les techniques d'entretien des locaux et du matériel :

- entretien quotidien de la chambre du patient (environnement) ; 
- bionettoyage chambre et chariot de chambre à la sortie du patient ;

- utiliser le matériel de brumisation;

- préparer une chambre en vue d'une entrée (tester le matériel d'aspiration, etc.) ;

- remplir le chariot de soins de la chambre ;

- remplir le poste avancé du couloir ;

- rechercher, traiter et transmettre les infos pour assurer la continuité des soins :

- organiser son travail en binôme avec l'IDE ;

- comprendre et respecter la sectorisation des patients selon leur statut (BMR/non BMR);

- savoir utiliser le dossier patient informatisé ;

- savoir utiliser les outils de soins : feuille de température informatisée, feuille de surveillance alimentaire, faire des transmissions écrites dans le dossier informatisé ;

- faire la commande des repas (en adéquation avec les régimes spécifiques éventuels ?) ;

- respecter les procédures d'isolement des patients ;

- connaître l'emplacement du chariot d'urgence, savoir l'acheminer sur le lieu de l'urgence ;

- savoir pratiquer un massage cardiaque externe ;

- connaître les numéros d'urgence ;

- utiliser les systèmes d'alerte en chambre ;

- inventaire patient ;

- physiopathologie des maladies les plus rencontrées et les prises en charge spécifiques de certains (caustiques, postop, caisson);

- pouvoir assister l'infirmière dans certains soins spécifiques de base.

Liens d'intérêts : les auteurs déclarent ne pas avoir de liens d'intérêts.

\section{Références}

1. Griffiths PD, Edwards MH, Forbes A, Harris RL, Ritchie G, (2007) Effectiveness of intermediate care in nursing-led inpatient units. http://cochranelibrary-wiley.com/doi/10.1002/ 14651858.CD002214.pub3/full (Dernier accès le 19 juin 2018)

2. Voultoury J, Pinsard M, Robert R, (2009) Unité de surveillance continue. Réanimation 17: 816-822

3. Sinuff T, Kahnamoui K, Cook DJ, Luce JM, Levy MM, Values Ethics and Rationing in Critical Care Task Force, (2004) Rationing critical care beds: a systematic review. Crit Care Med 32: $1588-1597$

4. République française, (2002) Décret $\mathrm{n}^{\mathrm{o}}$ 2002-466 du 5 avril 2002 relatif aux conditions techniques de fonctionnement auxquelles doivent satisfaire les établissements de santé pour pratiquer les activités de réanimation, de soins intensifs et de surveillance continue et modifiant le Code de la santé publique. https://www. legifrance.gouv.fr/affichTexte.do?cidTexte=JORFTEXT00000058 $5557 \&$ categorieLien $=$ cid (Dernier accès le 19 juin 2018)

5. Société de réanimation de langue française (SRLF), Société française d'anesthésie et de réanimation (SFAR), (2015) Recommanda- tions communes de la SRLF et de la SFAR portant sur la définition et le fonctionnement des unités de surveillance et de soins continus. http://www.srlf.org/wp-content/uploads/2015/12/200502Recommandations_SFAR_SRLF-OrganisationDesUSC.pdf (Dernier accès le 19 juin 2018)

6. Pearse RM, Moreno RP, Bauer P, Pelosi P, Metnitz P, Spies C, Vallet B, Vincent JL, Hoeft A, Rhodes A, European Surgical Outcomes Study (EuSOS) group for the Trials groups of the European Society of Intensive Care Medicine and the European Society of Anaesthesiology, (2012) Mortality after surgery in Europe: a 7 day cohort study. Lancet 380: 1059-1065

7. Gillies MA, Power GS, Harrison DA, Fleming A, Cook B, Walsh TS, Pearse RM, Rowan KM, (2015) Regional variation in critical care provision and outcome after high-risk surgery. Intensive Care Med 41: 1809-1816

8. Vlayen A, Verelst S, Bekkering GE, Schrooten W, Hellings J, Claes N, (2012) Incidence and preventability of adverse events requiring intensive care admission: a systematic review. J Eval Clin Pract 18: 485-497

9. Capuzzo M, Volta C, Tassinati T, Moreno R, Valentin A, Guidet B, Iapichino G, Martin C, Perneger T, Combescure C, Poncet A, Rhodes A, Working Group on Health Economics of the European Society of Intensive Care Medicine, (2014) Hospital mortality of adults admitted to Intensive Care Units in hospitals with and without Intermediate Care Units: a multicentre European cohort study. Crit Care 18: 551

10. Fitch K, Bernstein S, Aguilar M, Burnand B, LaCalle JR, Lazaro P, van het Loo M, McDonnell J, Vader J, Kahan JP, (2001) The RAND/UCLA appropriateness method user's manual. RAND, Santa Monica (CA)

11. Junker C, Zimmerman JE, Alzola C, Draper EA, Wagner DP, (2002) A multicenter description of intermediate-care patients: comparison with ICU low-risk monitor patients. Chest 121: $1253-1261$

12. Zimmerman JE, Wagner DP, Knaus WA, Williams JF, Kolakowski D, Draper EA, (1995) The use of risk predictions to identify candidates for intermediate care units. Implications for intensive care utilization and cost. Chest 108: 490-499

13. Beaussier MM, Boulkedid B, Baillard R, Guidet C, Aegerter B, Alberti PC, (2016) Critères d'éligibilité des patients en unités de surveillance continue : Enquête nationale de type Delphi (abstract). Ann Fr Anesth Reanim R517

14. Prin M, Wunsch H, (2014) The role of stepdown beds in hospital care. Am J Respir Crit Care Med 190: 1210-1216

15. Agence nationale d'appui à la performance (ANAP), (2011) Réanimation. Retour d'expérience sur les indicateurs de performance. http://www.anap.fr/publications-et-outils/publications/detail/ actualites/reanimation-retour-dexperiences-sur-des-indicateurs-deperformance/ (Dernier accès le 19 juin 2018)

16. Smith L, Orts CM, O’Neil I, Batchelor AM, Gascoigne AD, Baudouin SV, (1999) TISS and mortality after discharge from intensive care. Intensive Care Med 25: 1061-1065

17. Alban RF, Nisim AA, Ho J, Nishi GK, Shabot MM, (2006) Readmission to surgical intensive care increases severityadjusted patient mortality. J Trauma 60: 1027-1031

18. Jhanji S, Thomas B, Ely A, Watson D, Hinds CJ, Pearse RM, (2008) Mortality and utilisation of critical care resources amongst high-risk surgical patients in a large NHS trust. Anaesthesia 63: 695-700

19. Byrick RJ, Power JD, Ycas JO, Brown KA, (1986) Impact of an intermediate care area on ICU utilization after cardiac surgery. Crit Care Med 14: 869-872

20. Fox AJ, Owen-Smith O, Spiers P, (1999) The immediate impact of opening an adult high dependency unit on intensive care unit occupancy. Anaesthesia 54: 280-283 
21. Plesner LL, Iversen AK, Langkjær S, Nielsen TL, Østervig R, Warming PE, Salam IA, Kristensen M, Schou M, Eugen-Olsen J, Forberg JL, Køber L, Rasmussen LS, Sölétormos G, Pedersen BK, Iversen K, (2015) The formation and design of the TRIAGE study--baseline data on 6005 consecutive patients admitted to hospital from the emergency department. Scand J Trauma Resusc Emerg Med 23: 106

22. Davies J, Tamhane R, Scholefield C, Curley P, (1999) Does the introduction of HDU reduce surgical mortality? Ann R Coll Surg Engl 81: 343-347

23. Jones HJ, Coggins R, Lafuente J, de Cossart L, (1999) Value of a surgical high-dependency unit. Br J Surg 86: 1578-1582

24. McIlroy DR, Coleman BD, Myles PS, (2006) Outcomes following a shortage of high dependency unit beds for surgical patients. Anaesth Intensive Care 34: 457-463

25. Richards BF, Fleming JB, Shannon CN, Walters BC, Harrigan MR, (2012) Safety and cost effectiveness of step-down unit admission following elective neurointerventional procedures. J Neurointerv Surg 4: 390-392

26. Vester-Andersen M, Waldau T, Wetterslev J, Møller MH, Rosenberg J, Jørgensen LN, Jakobsen JC, Møller AM, InCare trial group, (2013) Randomized multicentre feasibility trial of intermediate care versus standard ward care after emergency abdominal surgery (InCare trial). Br J Surg 102: 619-629

27. Robert R, (2016) Admission en réanimation : critères et conséquences. In: Boles JM, Bollaert PE, Offenstadt G, Mercat A, Mira JP, Saulnier F, Wolff M, Zeni F (eds) Réanimation. Volume VI Section. Elsevier Masson, Paris, pp 387-388

28. Nasraway SA, Cohen IL, Dennis RC, Howenstein MA, Nikas DK, Warren J, Wedel SK, (1998) Guidelines on admission and discharge for adult intermediate care units. American College of Critical Care Medicine of the Society of Critical Care Medicine. Crit Care Med 26: 607-610

29. Matukaitis J, Stillman P, Wykpisz E, Ewen E, (2005) Appropriate admissions to the appropriate unit: a decision tree approach. Am J Med Qual 20: 90-97

30. Moonesinghe SR, Mythen MG, Das P, Rowan KM, Grocott MP, (2013) Risk stratification tools for predicting morbidity and mortality in adult patients undergoing major surgery: qualitative systematic review. Anesthesiology 119: 959-981

31. Moonesinghe SR, Mythen MG, Grocott MP, (2009) Patientrelated risk factors for postoperative adverse events. Curr Opin Crit Care 15: 320-327

32. American College of Cardiology/American Heart Association Task Force on Practice Guidelines (Writing Committee to Revise the 2002 Guidelines on Perioperative Cardiovascular Evaluation for Noncardiac Surgery), American Society of Echocardiography, American Society of Nuclear Cardiology, Heart Rhythm Society, Society of Cardiovascular Anesthesiologists, Society for Cardiovascular Angiography and Interventions, Society for Vascular Medicine and Biology, Society for Vascular Surgery, Fleisher LA, Beckman JA, Brown KA, Calkins H, Chaikof E, Fleischmann KE, Freeman WK, Froehlich JB, Kasper EK, Kersten JR,
Riegel B, Robb JF, Smith SC Jr, Jacobs AK, Adams CD, Anderson JL, Antman EM, Buller CE, Creager MA, Ettinger SM, Faxon DP, Fuster V, Halperin JL, Hiratzka LF, Hunt SA, Lytle BW, Nishimura R, Ornato JP, Page RL, Riegel B, Tarkington LG, Yancy CW, (2008) ACC/AHA 2007 guidelines on perioperative cardiovascular evaluation and care for noncardiac surgery: executive summary: a report of the American College of Cardiology/American Heart Association Task Force on Practice Guidelines (Writing Committee to Revise the 2002 Guidelines on Perioperative Cardiovascular Evaluation for Noncardiac Surgery). Anesth Analg 106: 685-712

33. Whiteley MS, Prytherch DR, Higgins B, Weaver PC, Prout WG, (1996) An evaluation of the POSSUM surgical scoring system. Br J Surg 83: 812-815

34. Junker C, Zimmerman JE, Alzola C, Draper EA, Wagner DP, (2002) A multicenter description of intermediate-care patients: comparison with ICU low-risk monitor patients. Chest 121: 1253-1261

35. Miranda DR, de Rijk A, Schaufeli W, (1996) Simplified therapeutic intervention scoring system: the TISS-28 items: results from a multicenter study. Crit Care Med 24: 64-73

36. Reis Miranda D, (1997) The Therapeutic Intervention Scoring System: one single tool for the evaluation of workload, the work process and management? Intensive Care Med 23: 615-617

37. Muehler N, Oishi J, Specht M Rissner F, Reinhart K, Sakr Y, (2010) Serial measurement of Therapeutic Intervention Scoring System-28 (TISS-28) in a surgical intensive care unit. J Crit Care 25: 620-627

38. Simpson JC, Moonesinghe SR, (2013) Introduction to the postanaesthetic care unit. Perioper Med 2: 5

39. Ministère de l'Économie et des Finances, ministère des Affaires sociales et de la Santé, (2017) Arrêté du 27 février 2017 modifiant l'arrêté du 19 février 2015 modifié relatif aux forfaits alloués aux établissements de santé mentionnés à l'article L. 162-22-6 du Code de la sécurité sociale (Dernier accès le 19 juin 2018)

40. Fourrier F, (2014) Structures et organisation des unités de surveillance continue (USC) 250 recommandations. Reanimation 23: $116-130$

41. Cheng DCH, Byrick RJ, Knobel E, (1999) Structural models for intermediate care areas. Crit Care Med 27: 2266-2271

42. Société de réanimation de langue française, Collège des réanimateurs extra-universitaires français, Groupe francophone de réanimation et urgences pédiatriques, Société française d'anesthésie et de réanimation, (2011) Référentiel de compétence de l'infirmière de réanimation. Réanimation 20: S737-S746

43. Société de réanimation de langue française, Collège des réanimateurs extra-universitaires français, Groupe francophone de réanimation et urgences pédiatriques, Société française d'anesthésie et de réanimation, (2011) Livret d'adaptation à l'emploi. Réanimation 20: S747-S766

44. République française, (2016) Décret $n^{\circ}$ 2016-524 du 27 avril 2016 relatif aux groupements hospitaliers de territoire. https:// www.legifrance.gouv.fr/eli/decret/2016/4/27/AFSH1609031D/jo (dernier accès le 19 juin 2018) 